\title{
Active Safety Control for Dynamic Human-Robot Interaction
}

\author{
Melanie Kimmel and Sandra Hirche \\ Chair of Information-Oriented Control \\ Technische Universität München \\ 80333 Munich, Germany \\ Email: melanie.kimmel@tum.de, hirche@tum.de
}

\begin{abstract}
In human-robot interaction (HRI) and especially in close or physical interaction, it is essential to ensure the human's safety. This is achieved by introducing virtual constraints defining a region, in which the robot is allowed to move safely. These safety regions may change over time during human-robot interaction, which may be either due to human motion or changed environmental conditions. In consequence it is important for the applied control scheme to handle dynamic boundaries. This work proposes an invariance-based control approach, which enforces adherence to boundaries with dynamic parameters. We extend the invariance control approach, which provides a computationally efficient and systematic method for defining constraints on system states and outputs, such that it handles the constraint dynamics. Stability and invariance properties are analyzed and validated in an experimental evaluation on a 7-DoF anthropomorphic manipulator.
\end{abstract}

\section{INTRODUCTION}

With the advances in research, robotic systems, are introduced in industrial and household settings. While it suffices to mount the system behind physical barriers to assure the safety of humans for some applications, other applications require close or physical interaction of humans and robots and therefore physical barriers are highly obstructive. The joint manipulation of an object, for example, requires the robot to move freely to assist a human partner in reaching a common goal. In order to enforce the safety of the human partner during the interaction, the robot motion has to be restricted by means of control. Additionally, as the constraints may change over time due to a dynamic environment and the moving human, control concepts have to be used, which are able to enforce dynamic constraints.

These requirements are closely related to the concept of virtual wall rendering. The most commonly used approaches, such as potential fields [1] and virtual fixtures [2], omit, however, explicit consideration of the system dynamics, such not being able to guarantee constraint satisfaction. Other possible approaches are found in constrained control. The most widely used control scheme is the optimization-based model predictive control [3]. It allows for input, state and output constraints but the application to nonlinear, dynamic systems with high dimension is computationally expensive, which may prevent application in real-time. A combination of control barrier functions with control Lyapunov functions [4] enables the simultaneous consideration of control objectives, hard and soft constraints on the system. The approach is, however, limited to time-invariant constraints. The reference governor approach [5] allows for system disturbances but the necessary numerical simulation may not fulfill real-time requirements. Invariance control is a promising alternative to the aforementioned approaches to realize active safety control. The invariance control approach deals with multiple constraints while following a nominal control goal whenever possible. Invariance control is designed for nonlinear, control affine single-input single-output (SISO) systems [6] but it is also applicable to multi-input multi-output (MIMO) systems [7]. The control scheme, which is based on input-output linearization (I/O-linearization) and a so-called invariance function, which takes the system dynamics into account, enables the implementation as an add-on to any existing stabilizing nominal control law, which enforces the control goal [8]. This makes it a promising approach for systems with an existing control structure, which are subject to safety issues. However, so far, invariance control is only provably safely applicable with static constraints.

In this work, we introduce an invariance control approach with dynamic constraints for a general robotic system with Lagrangian dynamics. It allows the design of the invariance controller as an add-on to an existing controller while also taking into account the dynamics of the boundaries. We investigate the stability of the controlled system. Furthermore, we analyse for which boundary dynamics, the system is rendered positively controlled invariant and any violation of the constraints is avoided. An experimental evaluation on an anthropomorphic manipulator with seven degrees of freedom (DoF) successfully validates the results.

The remainder of this paper is organized as follows: Section II introduces the basic principles of invariance control. Section III extends them to the use with robotic manipulators with dynamic constraints. Stability is investigated in Section IV and the invariance properties are discussed in Section V. Section VI illustrates the usefulness of impedance control with invariance control in HRI scenarios and section VII provides experimental results.

Notation: Bold small characters are used for vectors and bold capital characters for matrices. Matrix $\boldsymbol{A}=\left[\boldsymbol{a}_{i}^{\top}\right] \in$ $\mathbb{R}^{k \times n}$ and vector $\boldsymbol{b}=\left[b_{i}\right] \in \mathbb{R}^{k \times 1}$ are given by

$$
\boldsymbol{A}=\left[\boldsymbol{a}_{i}^{\top}\right]=\left[\begin{array}{c}
\boldsymbol{a}_{1}^{\top} \\
\vdots \\
\boldsymbol{a}_{k}^{\top}
\end{array}\right], \quad \boldsymbol{b}=\left[b_{i}\right]=\left[\begin{array}{c}
b_{1} \\
\vdots \\
b_{k}
\end{array}\right]
$$


The expression $\boldsymbol{x}_{1} \preccurlyeq \boldsymbol{x}_{2}$ denotes the element-wise inequality of two vectors $\boldsymbol{x}_{1}, \boldsymbol{x}_{2} \in \mathbb{R}^{n}$. The Euclidean vector norm (2norm) is abbreviated by $\|\boldsymbol{x}\|_{2}=\sqrt{\boldsymbol{x}^{\top} \boldsymbol{x}}$ for $\boldsymbol{x} \in \mathbb{R}^{n}$. Time derivatives of low order are abbreviated by dots $\dot{x}=\frac{\mathrm{d} x}{\mathrm{~d} t}$. The set $\mathcal{C}^{k}\left(\mathbb{R}^{m}, \mathbb{R}^{n}\right)$ denotes the set of $k$ times continuously differentiable functions with $h: \mathbb{R}^{n} \rightarrow \mathbb{R}^{m}$.

\section{General IdeA of InVARIANCE CONTROL}

This section gives a brief introduction to the steps of designing an invariance controller as an add-on to a nominal controller. More details are provided in [6]-[8].

Invariance control is used to enforce constraints on states and outputs of a dynamical system. It is designed as an addon to a nominal controller [8], resulting in the structure shown in Fig. 1. The nominal controller is designed to

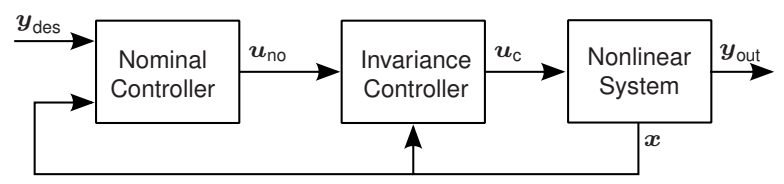

Fig. 1: Structure of an invariance controlled system [7]

achieve a specific control goal, e.g. trajectory tracking and to stabilize the system in the sense of Lyapunov. The invariance controller monitors the system outputs and states with respect to the defined constraints and changes the control output only if it is necessary to avoid a violation. Invariance control is applicable to nonlinear, control affine MIMO systems [7]

$$
\left.\begin{array}{l}
\dot{\boldsymbol{x}}=\boldsymbol{f}(\boldsymbol{x})+\boldsymbol{G}(\boldsymbol{x}) \boldsymbol{u} \\
\boldsymbol{y}_{\text {out }}=\boldsymbol{h}_{\text {out }}(\boldsymbol{x})
\end{array}\right\}
$$

with the states $\boldsymbol{x} \in \mathbb{R}^{n}$, the input $\boldsymbol{u} \in \mathcal{U}_{\text {no }} \subset \mathbb{R}^{m}$, the output function vector $\boldsymbol{h}_{\text {out }}: \mathbb{R}^{n} \rightarrow \mathbb{R}^{q}$, and sufficiently smooth vector functions $f: \mathbb{R}^{n} \rightarrow \mathbb{R}^{n}, \boldsymbol{g}_{i}: \mathbb{R}^{n} \rightarrow \mathbb{R}^{n}$ such that $\boldsymbol{G}=\left[\boldsymbol{g}_{1} \ldots \boldsymbol{g}_{m}\right]$. The design steps for single input and/or single output systems are the same as for MIMO systems.

The element-wise inequality of the output function vector

$$
\boldsymbol{y}=\boldsymbol{h}(\boldsymbol{x}, \boldsymbol{\eta}) \preccurlyeq 0,
$$

with $\boldsymbol{y} \in \mathbb{R}^{l}$ and the constant parameters $\boldsymbol{\eta} \in \mathbb{R}^{n_{\eta}}$ defines $l$ constraints, where $l$ may be an arbitrarily large number [9]. This means that invariance control allows for arbitrarily many constraints. Thus it is applicable in scenarios involving multiple humans/obstacles and it may also avoid collision with the entire volume of the robotic system. Each constraint $i$ is defined by one output function $y_{i}=h_{i}(\boldsymbol{x}, \boldsymbol{\eta})$, which has to be $r_{i}$ times continuously differentiable, i.e. $h_{i}(\boldsymbol{x}, \boldsymbol{\eta}) \in$ $\mathcal{C}^{r_{i}}\left(\mathbb{R}^{n}, \mathbb{R}\right)$, where $r_{i}$ is the relative degree of the system (1) resulting from the I/O-linearization of the system with respect to the output function $h_{i}(\boldsymbol{x}, \boldsymbol{\eta})$. The output function equals zero on the constraint and takes a negative value inside the admissible set

$$
\mathcal{H}=\left\{\boldsymbol{x} \in \mathbb{R}^{n} \mid h_{i}(\boldsymbol{x}, \boldsymbol{\eta}) \leq 0 \quad \forall 1 \leq i \leq l\right\} .
$$

The constraints depend on the system states and fixed boundary parameters. They are chosen such that the admissible set is non-empty.

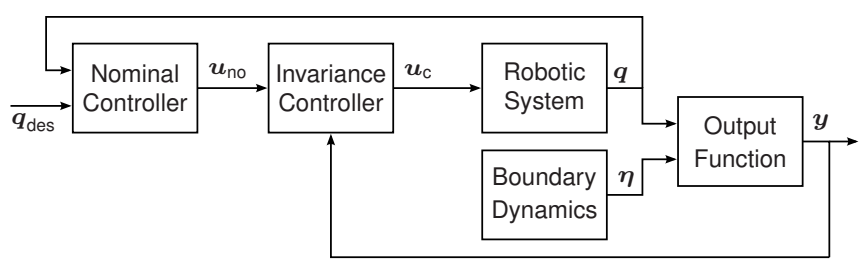

Fig. 2: Structure of an invariance controlled system with dynamic boundaries

The so-called invariance functions $\Phi_{i}\left(\boldsymbol{x}, \boldsymbol{\eta}, \gamma_{i}\right)$ extend the concept of the output functions by taking the dynamics of the system (1) into account. They define the invariant set

$$
\mathcal{G}=\left\{\boldsymbol{x} \in \mathbb{R}^{n} \mid \Phi_{i}\left(\boldsymbol{x}, \gamma_{i}\right) \leq 0 \quad \forall 1 \leq i \leq l\right\} .
$$

The goal of invariance control is to determine a control output $\boldsymbol{u}_{\mathrm{c}}$, which renders the system (1) controlled positive invariant with respect to the invariant set (4). This means that once the set is entered, the system states will remain therein for all future times. For a robotic system with static constraints, the design of an invariance controller is described in [7], [10]. This approach is useful if the robot is mounted in an environment with static obstacles and the workspaces of human and robot are strictly separated. In dynamic environments or for tasks with overlapping workspaces, it is, however, not possible to describe the boundaries using static constraints. Therefore, we introduce an invariance control approach for robotic systems with dynamic boundaries.

\section{Robotic Systems With DyNAMIC Boundaries}

We consider a robotic system with generalized dynamics

$$
M_{\boldsymbol{q}}(\boldsymbol{q}) \ddot{\boldsymbol{q}}+\boldsymbol{C}_{\boldsymbol{q}}(\boldsymbol{q}, \dot{\boldsymbol{q}}) \dot{\boldsymbol{q}}+\boldsymbol{g}_{\boldsymbol{q}}(\boldsymbol{q})=\boldsymbol{\tau}
$$

with the generalized coordinates $\boldsymbol{q}(t) \in \mathbb{R}^{n_{q}}$, the mass matrix $\boldsymbol{M}_{\boldsymbol{q}}(\boldsymbol{q}) \in \mathbb{R}^{n_{q} \times n_{q}}$, the Coriolis forces $\boldsymbol{C}_{\boldsymbol{q}}(\boldsymbol{q}, \dot{\boldsymbol{q}}) \dot{\boldsymbol{q}} \in \mathbb{R}^{n_{q}}$, the gravitational effects $\boldsymbol{g}_{\boldsymbol{q}}(\boldsymbol{q}) \in \mathbb{R}^{n_{q}}$ and the input $\boldsymbol{\tau} \in \mathbb{R}^{n_{q}}$. For notational convenience, the explicit dependency on $\boldsymbol{q}$ of the matrix $M_{\boldsymbol{q}}(\boldsymbol{q})$ will be omitted in the following. A transformation into the form of (1) yields

$$
\underbrace{\left(\begin{array}{c}
\dot{\boldsymbol{q}} \\
\ddot{\boldsymbol{q}}
\end{array}\right)}_{\dot{\boldsymbol{x}}}=\underbrace{\left(\begin{array}{c}
\dot{\boldsymbol{q}} \\
-M_{\boldsymbol{q}}^{-1}\left(\boldsymbol{C}_{\boldsymbol{q}}(\boldsymbol{q}, \dot{\boldsymbol{q}}) \dot{\boldsymbol{q}}+\boldsymbol{g}_{\boldsymbol{q}}(\boldsymbol{q})\right)
\end{array}\right)}_{\boldsymbol{f}(\boldsymbol{x})}+\underbrace{\left(\begin{array}{c}
0 \\
M_{\boldsymbol{q}}^{-1}
\end{array}\right)}_{\boldsymbol{G}(\boldsymbol{x})} u
$$

with $\boldsymbol{x}=\left[\boldsymbol{q}^{\top}, \dot{\boldsymbol{q}}^{\top}\right]^{\top}$ and $\boldsymbol{u}=\boldsymbol{\tau}$. The structure of the controlled system with dynamic parameters is shown in Fig. 2. The nonlinear, robotic system (6) is controlled by a nominal control output $\boldsymbol{u}_{\text {no }}$, which stabilizes the system in the sense of Lyapunov. Corrective control $\boldsymbol{u}_{\mathrm{c}}$ is generated by the invariance control scheme by combining the nominal control output with the constraint information.

\section{A. Dynamic Environments}

The dynamic constraints are specified by a vector of dynamic output functions

$$
\boldsymbol{h}(\boldsymbol{x}(t), \boldsymbol{\eta}(t)) \preccurlyeq \mathbf{0}
$$


with $\boldsymbol{h}(\boldsymbol{x}(t), \boldsymbol{\eta}(t)) \in \mathbb{R}^{l}$, which depend on the system states $\boldsymbol{x}(t)$ and the dynamic parameters $\boldsymbol{\eta}(t)$. The vector defines the dynamically changing admissible set

$$
\mathcal{H}(t)=\left\{\boldsymbol{x} \in \mathbb{R}^{n} \mid h_{i}(\boldsymbol{x}(t), \boldsymbol{\eta}(t)) \leq 0 \quad \forall 1 \leq i \leq l\right\} .
$$

Since the goal of invariance control is to keep the system (6) within the admissible set, the admissible set $\mathcal{H}$ (3) may at no instant of time equal the empty set

$$
\mathcal{H} \neq\{\} \quad \forall t>0 .
$$

In the following, for lack of space, we consider a vector of output functions $\boldsymbol{h}(\boldsymbol{q}(t), \boldsymbol{\eta}(t))$ independent from $\dot{\boldsymbol{q}}$ representing e.g. constraints in the task space. Velocity dependent constraints, i.e. output functions with an explicit dependency on $\dot{\boldsymbol{q}}$, are also straightforwardly implementable. This only changes the relative degree and the invariance function [8] but the general control design steps remain unchanged.

\section{B. Input/Output (I/O)-Linearization}

In order to design of invariance control as an add-on to a nominal control scheme and to determine the invariance function, I/O-linearization of the system (6) with respect to the output function (7) is performed [8]. Differentiation with respect to time

$$
\begin{aligned}
\dot{\boldsymbol{y}} & =\frac{\partial \boldsymbol{h}}{\partial \boldsymbol{q}} \dot{\boldsymbol{q}}+\frac{\partial \boldsymbol{h}}{\partial \boldsymbol{\eta}} \dot{\boldsymbol{\eta}}, \\
\ddot{\boldsymbol{y}} & =\frac{\partial \dot{\boldsymbol{y}}}{\partial \dot{\boldsymbol{q}}} \ddot{\boldsymbol{q}}+\frac{\partial \dot{\boldsymbol{y}}}{\partial \boldsymbol{q}} \dot{\boldsymbol{q}}+\frac{\partial \dot{\boldsymbol{y}}}{\partial \boldsymbol{\eta}} \dot{\boldsymbol{\eta}}+\frac{\partial \dot{\boldsymbol{y}}}{\partial \dot{\boldsymbol{\eta}}} \ddot{\boldsymbol{\eta}} \\
= & \frac{\partial \boldsymbol{h}}{\partial \boldsymbol{q}} \ddot{\boldsymbol{q}}+\frac{\partial}{\partial \boldsymbol{q}}\left(\frac{\partial \boldsymbol{h}}{\partial \boldsymbol{q}} \dot{\boldsymbol{q}}\right) \dot{\boldsymbol{q}}+2 \frac{\partial}{\partial \boldsymbol{\eta}}\left(\frac{\partial \boldsymbol{h}}{\partial \boldsymbol{q}} \dot{\boldsymbol{q}}\right) \dot{\boldsymbol{\eta}} \\
& +\frac{\partial}{\partial \boldsymbol{\eta}}\left(\frac{\partial \boldsymbol{h}}{\partial \boldsymbol{\eta}} \dot{\boldsymbol{\eta}}\right) \dot{\boldsymbol{\eta}}+\frac{\partial \boldsymbol{h}}{\partial \boldsymbol{\eta}} \ddot{\boldsymbol{\eta}} \\
= & \frac{\partial \boldsymbol{h}}{\partial \boldsymbol{q}} \boldsymbol{M}_{\boldsymbol{q}}{ }^{-1} \boldsymbol{u}-\frac{\partial \boldsymbol{h}}{\partial \boldsymbol{q}} \boldsymbol{M}_{\boldsymbol{q}}{ }^{-1}\left(\boldsymbol{C}_{\boldsymbol{q}}(\boldsymbol{q}, \dot{\boldsymbol{q}}) \dot{\boldsymbol{q}}+\boldsymbol{g}_{\boldsymbol{q}}(\boldsymbol{q})\right) \\
& +\frac{\partial}{\partial \boldsymbol{q}}\left(\frac{\partial \boldsymbol{h}}{\partial \boldsymbol{q}} \dot{\boldsymbol{q}}\right) \dot{\boldsymbol{q}}+2 \frac{\partial}{\partial \boldsymbol{\eta}}\left(\frac{\partial \boldsymbol{h}}{\partial \boldsymbol{q}} \dot{\boldsymbol{q}}\right) \dot{\boldsymbol{\eta}} \\
& +\frac{\partial}{\partial \boldsymbol{\eta}}\left(\frac{\partial \boldsymbol{h}}{\partial \boldsymbol{\eta}} \dot{\boldsymbol{\eta}}\right) \dot{\boldsymbol{\eta}}+\frac{\partial \boldsymbol{h}}{\partial \boldsymbol{\eta}} \ddot{\boldsymbol{\eta}}
\end{aligned}
$$

yields the I/O-linearizing input of (6)

$$
\boldsymbol{z}=\ddot{\boldsymbol{y}}=\boldsymbol{A}(\boldsymbol{x}, \boldsymbol{\eta}) \boldsymbol{u}+\boldsymbol{b}(\boldsymbol{x}, \boldsymbol{\eta})+\boldsymbol{\beta}(\boldsymbol{x}, \boldsymbol{\eta}, \dot{\boldsymbol{\eta}}, \ddot{\boldsymbol{\eta}})
$$

with $\boldsymbol{x}$ from (6), the pseudo input $\boldsymbol{z}$, which is the input to the integrator chain resulting from the I/O-linearization, and

$$
\begin{aligned}
\boldsymbol{A}(\boldsymbol{x}, \boldsymbol{\eta})= & \frac{\partial \boldsymbol{h}}{\partial \boldsymbol{q}} \boldsymbol{M}_{\boldsymbol{q}}^{-1} \\
\boldsymbol{b}(\boldsymbol{x}, \boldsymbol{\eta})= & -\frac{\partial \boldsymbol{h}}{\partial \boldsymbol{q}} \boldsymbol{M}_{\boldsymbol{q}}^{-1}\left(\boldsymbol{C}_{\boldsymbol{q}}(\boldsymbol{q}, \dot{\boldsymbol{q}}) \dot{\boldsymbol{q}}+\boldsymbol{g}_{\boldsymbol{q}}(\boldsymbol{q})\right) \\
& \quad+\frac{\partial}{\partial \boldsymbol{q}}\left(\frac{\partial \boldsymbol{h}}{\partial \boldsymbol{q}} \dot{\boldsymbol{q}}\right) \dot{\boldsymbol{q}} \\
\boldsymbol{\beta}(\boldsymbol{x}, \boldsymbol{\eta}, \dot{\boldsymbol{\eta}}, \ddot{\boldsymbol{\eta}})= & \frac{\partial}{\partial \boldsymbol{\eta}}\left(2 \frac{\partial \boldsymbol{h}}{\partial \boldsymbol{q}} \dot{\boldsymbol{q}}+\frac{\partial \boldsymbol{h}}{\partial \boldsymbol{\eta}} \dot{\boldsymbol{\eta}}\right) \dot{\boldsymbol{\eta}}+\frac{\partial \boldsymbol{h}}{\partial \boldsymbol{\eta}} \ddot{\boldsymbol{\eta}}
\end{aligned}
$$

If $\frac{\partial h_{i}}{\partial \boldsymbol{q}} \neq \mathbf{0}$ and $\frac{\partial h_{i}}{\partial \boldsymbol{q}} \boldsymbol{M}_{\boldsymbol{q}}{ }^{-1} \neq \mathbf{0} \forall i$ holds, each row of $\boldsymbol{A}(\boldsymbol{x}, \boldsymbol{\eta})$ has at least one non-zero element. Then the relative degree of each constraint $h_{i}(\boldsymbol{q}, \boldsymbol{\eta})$ is well-defined and $r_{i}=2$. It is also observed that $\boldsymbol{A}(\boldsymbol{x}, \boldsymbol{\eta})$ and $\boldsymbol{b}(\boldsymbol{x}, \boldsymbol{\eta})$ are independent from the time derivatives $\dot{\eta}, \ddot{\eta}$ of the boundary dynamics. This means that they have the same structure as in the time-invariant case. Only the additional term $\boldsymbol{\beta}(\boldsymbol{x}, \boldsymbol{\eta}, \dot{\boldsymbol{\eta}}, \ddot{\boldsymbol{\eta}})$, which is equal to zero in the timeinvariant case ( $\dot{\boldsymbol{\eta}}=0$ and $\ddot{\boldsymbol{\eta}}=0$ ), describes the influence of the parameter dynamics on the system.

Note that this approach is valid for any constraints defined in generalized coordinates $q$ as well as constraints defined in task space coordinates $\boldsymbol{p} \in \mathbb{R}^{n_{p}}$ as long as the transformation

$$
p=f_{p}(q)
$$

is known. In this case,

$$
\frac{\partial h_{i}}{\partial \boldsymbol{q}}=\frac{\partial h_{i}}{\partial \boldsymbol{p}} \frac{\partial \boldsymbol{p}}{\partial \boldsymbol{q}}=\frac{\partial h_{i}}{\partial \boldsymbol{p}} \boldsymbol{J}(\boldsymbol{q}) \neq 0
$$

has to hold, i.e. the Jacobian $\boldsymbol{J}(\boldsymbol{q})$ has full rank and $\frac{\partial h_{i}}{\partial \boldsymbol{p}}$ does not lie in the null-space of the Jacobian. This means that the manipulator is able to carry out movements in arbitrary directions and especially away from the constraint.

Invariance control assures that the system input $\boldsymbol{u}$ is equal to the nominal control output $\boldsymbol{u}_{\text {no }}$, whenever the system is at a distance from the constraints. Otherwise, a corrective control output is determined. The necessity of applying corrective control is determined by the invariance function.

\section{Invariance Function}

The invariance functions are given by

$$
\Phi_{i}\left(\boldsymbol{x}, \boldsymbol{\eta}, \gamma_{i}\right)= \begin{cases}-\frac{1}{2 \gamma_{i}} \dot{y}_{i}^{2}+y_{i} & \dot{y}_{i}>0 \\ y_{i} & \dot{y}_{i} \leq 0,\end{cases}
$$

since the relative degrees $r_{i}$ are equal to two [8]. The invariance functions for the robotic manipulator combine the output functions of the constraints with the knowledge of the system dynamics (6). A value $\Phi_{i}\left(\boldsymbol{x}, \boldsymbol{\eta}, \gamma_{i}\right)=0$ determines, when the manipulator moves at a velocity and is in a configuration such that applying a corrective counteracceleration will just avoid a violation of the constraint.

The system (6) is rendered controlled positive invariant with respect to (4), if one of the conditions

$$
\begin{aligned}
y_{i}^{(r)}(\boldsymbol{x}, \boldsymbol{\eta}) & <0 \quad \forall 1 \leq r \leq r_{i}-1 \\
y_{i}^{\left(r_{i}\right)}(\boldsymbol{x}, \boldsymbol{\eta}) & \leq \gamma_{i}
\end{aligned}
$$

is fulfilled [8], i.e. if either the system dynamics are directed away from the constraint or $z_{i} \leq \gamma_{i}<0$ is applied, which counteracts the system dynamics to avoid boundary violation. This motivates the design of the invariance control law.

\section{Corrective Control}

Corrective control action is only necessary for constraints, which are in danger of being violated. Therefore, the set of active constraints

$$
\mathcal{K}=\left\{i \in\{1,2, \ldots, l\} \mid \Phi_{i} \geq 0\right\}
$$


is introduced [7]. Nominal control is sufficient for constraints, which are not active.

With (11), (16) and (17), the element-wise condition for the corrective control output $\boldsymbol{u}_{\mathrm{c}}$ is given based on [7]

$$
\boldsymbol{A}_{\mathcal{K}}(\boldsymbol{x}, \boldsymbol{\eta}) \boldsymbol{u}_{\mathrm{c}}+\boldsymbol{b}_{\mathcal{K}}(\boldsymbol{x}, \boldsymbol{\eta})+\boldsymbol{\beta}_{\mathcal{K}}(\boldsymbol{x}, \boldsymbol{\eta}, \dot{\boldsymbol{\eta}}, \ddot{\boldsymbol{\eta}}) \preccurlyeq \gamma .
$$

with $\boldsymbol{A}_{\mathcal{K}}(\boldsymbol{x}, \boldsymbol{\eta}), \boldsymbol{b}_{\mathcal{K}}(\boldsymbol{x}, \boldsymbol{\eta}), \boldsymbol{\beta}_{\mathcal{K}}(\boldsymbol{x}, \boldsymbol{\eta}, \dot{\boldsymbol{\eta}}, \ddot{\boldsymbol{\eta}})$ being reduced forms of the matrix and vectors from (11), where the rows $i$ corresponding to inactive constraints $i \notin \mathcal{K}$ are removed and the vector $\gamma=\left[\gamma_{j}\right]$ with $j \in \mathcal{K}$.

Solving the constrained minimization problem

$$
\begin{gathered}
\min _{\boldsymbol{u}_{\mathrm{c}}}\left\|\boldsymbol{u}_{\mathrm{c}}-\boldsymbol{u}_{\mathrm{no}}\right\|_{2}^{2} \\
\text { s.t. } \boldsymbol{A}_{\mathcal{K}}(\boldsymbol{x}, \boldsymbol{\eta}) \boldsymbol{u}_{\mathrm{c}}+\boldsymbol{b}_{\mathcal{K}}(\boldsymbol{x}, \boldsymbol{\eta})+\boldsymbol{\beta}_{\mathcal{K}}(\boldsymbol{x}, \boldsymbol{\eta}, \dot{\boldsymbol{\eta}}, \ddot{\boldsymbol{\eta}}) \preccurlyeq \gamma
\end{gathered}
$$

then yields the corrective control output which is, in the sense of the Euclidean distance, closest to the nominal control output. Similar to [7], if the number of active constraints is smaller than the input dimension $|\mathcal{K}| \leq \operatorname{dim}(\boldsymbol{u})$, an analytical solution of (19) is given by

$$
\begin{aligned}
\boldsymbol{u}_{\mathrm{c}}= & \boldsymbol{A}_{\mathcal{K}}^{+}\left(\boldsymbol{z}_{\mathrm{c}}-(\underbrace{\boldsymbol{A}_{\mathcal{K}} \boldsymbol{u}_{\mathrm{no}}+\boldsymbol{b}_{\mathcal{K}}+\boldsymbol{\beta}_{\mathcal{K}}}_{\boldsymbol{z}_{\mathrm{no}}})\right)+\boldsymbol{u}_{\mathrm{no}} \\
= & \left(\frac{\partial \boldsymbol{h}_{\mathcal{K}}}{\partial \boldsymbol{q}} \boldsymbol{M}_{\boldsymbol{q}}{ }^{-1}\right)^{+}\left(\boldsymbol{z}_{\mathrm{c}}-\left(\frac{\partial \boldsymbol{h}_{\mathcal{K}}}{\partial \boldsymbol{q}} \boldsymbol{M}_{\boldsymbol{q}}{ }^{-1} \boldsymbol{u}_{\mathrm{no}}\right.\right. \\
& -\frac{\partial \boldsymbol{h}_{\mathcal{K}}}{\partial \boldsymbol{q}} \boldsymbol{M}_{\boldsymbol{q}}{ }^{-1}\left(\boldsymbol{C}_{\boldsymbol{q}}(\boldsymbol{q}, \dot{\boldsymbol{q}}) \dot{\boldsymbol{q}}+\boldsymbol{g}_{\boldsymbol{q}}(\boldsymbol{q})\right) \\
& +\frac{\partial}{\partial \boldsymbol{q}}\left(\frac{\partial \boldsymbol{h}_{\mathcal{K}}}{\partial \boldsymbol{q}} \dot{\boldsymbol{q}}\right) \dot{\boldsymbol{q}}+\frac{\partial}{\partial \boldsymbol{\eta}}\left(2 \frac{\partial \boldsymbol{h}_{\mathcal{K}}}{\partial \boldsymbol{q}} \dot{\boldsymbol{q}}\right. \\
& \left.\left.\left.+\frac{\partial \boldsymbol{h}_{\mathcal{K}}}{\partial \boldsymbol{\eta}} \dot{\boldsymbol{\eta}}\right) \dot{\boldsymbol{\eta}}+\frac{\partial \boldsymbol{h}_{\mathcal{K}}}{\partial \boldsymbol{\eta}}\right)\right)+\boldsymbol{u}_{\mathrm{no}}
\end{aligned}
$$

with the corrective pseudo input

$$
z_{\mathrm{c}, i}= \begin{cases}\gamma_{i} & \text { if }\left(z_{\mathrm{no}, i}>\gamma_{i}\right) \wedge\left(\Phi_{i} \geq 0\right) \\ z_{\mathrm{no}, i} & \text { if }\left(z_{\mathrm{no}, i} \leq \gamma_{i}\right) \vee\left(\Phi_{i}<0\right) .\end{cases}
$$

and the Moore-Penrose pseudo inverse $\boldsymbol{A}_{\mathcal{K}}^{+}=\boldsymbol{A}_{\mathcal{K}}^{\top}\left(\boldsymbol{A}_{\mathcal{K}} \boldsymbol{A}_{\mathcal{K}}^{\top}\right)$. This control law renders a system with time-invariant boundary parameters provably invariant with respect to the constraints [7]. It remains to show that this is also true for dynamic boundary parameters and that the invariance control law does not destabilize the nominally controlled system.

\section{Stability}

As illustrated in Fig. 2, the nominal control law is independent from the constraints on the system, which means that with a stabilizing nominal control law, the system under nominal control is stable in the sense of Lyapunov.

However, possibly unstable internal dynamics occur after I/O-linearization of the system (11). Assuming that the system (6) with static constraints has internal dynamics, which are stable in the sense of Lyapunov, it remains to show, that the dynamic change of the parameters does not cause a destabilization of the internal dynamics.

The I/O-linearization (11) transforms the system into $|\mathcal{K}|$ integrator chains with the output functions (7) as outputs, the inputs $z_{i}$ and the states $\boldsymbol{\xi}_{i} \in \mathbb{R}^{r_{i}}$ and the internal dynamics,

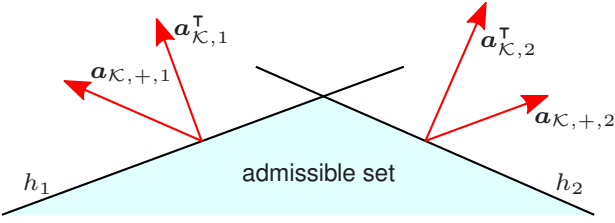

Fig. 3: Illustration of the relation between the rows $\boldsymbol{a}_{\mathcal{K}, i}^{\top}$ of $\boldsymbol{A}_{\mathcal{K}}$ and the columns $\boldsymbol{a}_{\mathcal{K},+, i}$ of $\boldsymbol{A}_{\mathcal{K}}^{+}$for a system with two constraints $h_{i}, i \in\{1,2\}$

which are not observable at the outputs and not influenced by the inputs $z_{i}$ [11]. The number of states of the internal dynamics $\boldsymbol{\xi}_{\text {int }} \in \mathbb{R}^{n_{\text {int }}}$ is determined by the number of states of (6) and the relative degrees $r_{i}$. It is given by $n_{\text {int }}=n-r$ with $r=\sum_{i} r_{i}$. Therefore, the states of the I/O-linearized system are given by the (local) diffeomorphism [12, pp. 515]

$$
\left(\begin{array}{c}
\boldsymbol{\xi}_{\text {int }} \\
\boldsymbol{\xi}
\end{array}\right)=\left(\begin{array}{c}
\boldsymbol{\Psi}(\boldsymbol{x}, \boldsymbol{\eta}) \\
{\left[\boldsymbol{\xi}_{i}\right]}
\end{array}\right)
$$

The relation between the states of the internal dynamics $\boldsymbol{\xi}_{\text {int }}$ and the states $\boldsymbol{x}$ of the original system (6) depends on the choice of the output functions $\boldsymbol{h}(\boldsymbol{x}, \boldsymbol{\eta})$. It is determined by the transformation $\Psi(\boldsymbol{x}, \boldsymbol{\eta})$, which fulfills [12, pp. 515]

$$
\frac{\partial \boldsymbol{\Psi}(\boldsymbol{x}, \boldsymbol{\eta})}{\partial \boldsymbol{x}} \boldsymbol{G}(\boldsymbol{x})=\mathbf{0} .
$$

Since the output function (7) shows no explicit dependence on the dynamics of the boundary parameters $\dot{\eta}$ and $\ddot{\eta}$, the transformation $\Psi(\boldsymbol{x}, \boldsymbol{\eta})$ is independent from the boundary dynamics. In consequence, the internal dynamics of the I/Olinearized system are the same for constraints with dynamic parameters as for constant parameters and a dynamic change of parameters does not destabilize the internal dynamics.

With stable internal dynamics and a stabilizing nominal controller, the invariance properties of the invariance controlled system have to be examined.

\section{INVARIANCE PROPERTIES}

For the analysis of the invariance properties, we assume, that the number of constraints in the task space is limited and arranged such that the number of active constraints is less then the input dimension, i.e. $\operatorname{dim}(u) \geq|\mathcal{K}|$. The corrective control (20) is re-written in the form

$$
\boldsymbol{u}_{\mathrm{c}}=\boldsymbol{A}_{\mathcal{K}}^{+}\left(\boldsymbol{z}_{\mathrm{c}}-\boldsymbol{b}_{\mathcal{K}}-\boldsymbol{\beta}_{\mathcal{K}}\right)+\left(\boldsymbol{I}-\boldsymbol{A}_{\mathcal{K}}^{+} \boldsymbol{A}_{\mathcal{K}}\right) \boldsymbol{u}_{\mathrm{no}} .
$$

The term $\left(\boldsymbol{I}-\boldsymbol{A}_{\mathcal{K}}^{+} \boldsymbol{A}_{\mathcal{K}}\right) \boldsymbol{u}_{\text {no }}$ has no influence on the value of the output and the invariance functions, since $\left(\boldsymbol{I}-\boldsymbol{A}_{\mathcal{K}}^{+} \boldsymbol{A}_{\mathcal{K}}\right)$ projects the nominal control output into the null-space of the constraints [13]. Due to $\boldsymbol{A}_{\mathcal{K}} \boldsymbol{A}_{\mathcal{K}}^{+}=\boldsymbol{I}$, each column vector of $\boldsymbol{A}_{\mathcal{K}}^{+}$is directed out of the admissible set of one constraint and is invariant with respect to the other constraints as depicted in Fig. 3. For a constraint admissible control output, each column of $\boldsymbol{A}_{\mathcal{K}}^{+}$has to be multiplied by a non-positive number, which is achieved by the element-wise condition

$$
\boldsymbol{z}_{\mathrm{c}}-\boldsymbol{b}_{\mathcal{K}}-\boldsymbol{\beta}_{\mathcal{K}} \preccurlyeq 0
$$

which has to hold for all times. Since $z_{\mathrm{c}, i}$ is set to a constant value $\gamma_{i}(21)$, the term $\boldsymbol{b}_{\mathcal{K}}+\boldsymbol{\beta}_{\mathcal{K}}$, which depends 
on the dynamic constraint parameters, has to be bounded. This term depends on the parameters $\boldsymbol{\eta}(t)$ and their time derivatives up to the $r_{i}$-th order, which follows from (11). Therefore, the system (6) is rendered positive invariant with respect to the constraints with dynamic parameters $\boldsymbol{\eta}(t)$, if the constraint parameters $\boldsymbol{\eta}(t)$ are bounded and $r_{i}$-times continuously differentiable with bounded derivatives such that (25) is fulfilled for a constant $z_{\mathrm{c}}=\gamma$ for all times.

\section{INVARIANCE AND IMPEDANCE CONTROL}

Impedance control is a widely used control law in HRI for tasks. In contrast to position control, it allows the manipulator to react compliantly to exerted forces, such increasing the safety of the interacting human. In task space coordinates $\boldsymbol{p}$, the impedance control law [14] is given by

$$
\boldsymbol{u}_{\mathrm{no}}=\boldsymbol{f}_{\mathrm{ext}}+\boldsymbol{D}_{\boldsymbol{p}}\left(\dot{\boldsymbol{p}}_{\mathrm{des}}-\boldsymbol{J}(\boldsymbol{q}) \dot{\boldsymbol{q}}\right)+\boldsymbol{K}_{\boldsymbol{p}}\left(\boldsymbol{p}_{\mathrm{des}}-\boldsymbol{f}_{\boldsymbol{p}}(\boldsymbol{q})\right)
$$

with $\boldsymbol{p}=\boldsymbol{f}_{\boldsymbol{p}}(\boldsymbol{q})$ and the Jacobian $\boldsymbol{J}(\boldsymbol{q})=\frac{\partial \boldsymbol{p}}{\partial \boldsymbol{q}}$. It imitates spring $\boldsymbol{K}_{\boldsymbol{p}} \in \mathbb{R}^{n_{p} \times n_{p}}$ damper $\boldsymbol{D}_{\boldsymbol{p}} \in \mathbb{R}^{n_{p} \times n_{p}}$ behavior in reaction to an external force $\boldsymbol{f}_{\text {ext }} \in \mathbb{R}^{n_{p}}$ and a desired trajectory $\boldsymbol{p}_{\text {des }}$. In order to enhance the safety during the interaction, impedance control is combined with invariance control. In the combined control scheme, impedance control serves as the nominal controller. We distinguish two types of HRI scenarios in a dynamic environment for which this combination is useful.

In the first scenario human and robot work in (close) proximity without physical contact. Exemplary applications include domestic scenarios such as jointly laying out the table or industrial applications such as assembling tasks. Here $\boldsymbol{f}_{\text {ext }}$ equals zero due to the lack of physical contact. The robot carries out a desired, goal-directed motion. The constraints enforced by the invariance control scheme have to represent the limits imposed by the moving human and the dynamic environment. Then the manipulator carries out its task as long as it is safe but as soon as the human or an obstacle approaches, its motion is restricted.

In the second scenario human and robot are coupled, either by direct contact or through an object. Both try to reach a common goal and the human may exert forces, i.e. $\boldsymbol{f}_{\text {ext }} \neq 0$. An exemplary application is the joint manipulation of large objects. The robot assists the human in carrying the object and the impedance control scheme allows the human to exert forces to adjust the motion of the object. In addition to adjusting the trajectory of the robot if a constraint approaches, here, the invariance control scheme ensures that even if forces are exerted, which push the manipulator towards a boundary, no violation occurs. As the corrective control output replaces the nominal impedance controller, the manipulator shows stiff behavior at the constraint instead of reacting compliantly to forces.

In both scenarios, possibly including multiple humans and/or robots, impedance control with invariance control results in a safer interaction. The strict adherence to constraints is guaranteed even in the presence of external forces and in dynamic environments and the common interaction goal is achieved to the extent to which the limits permit it.

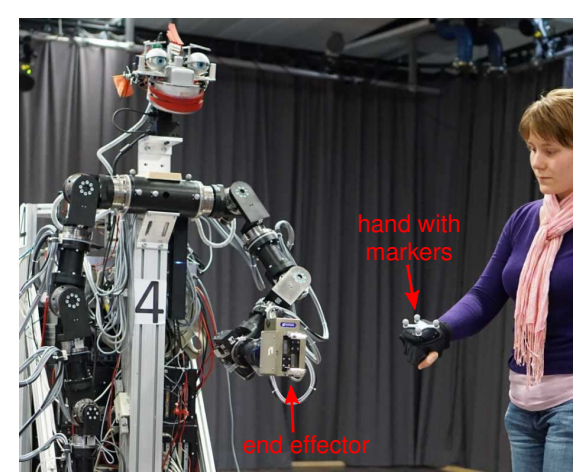

Fig. 4: Experimental procedure: The human hand with markers is detected and defines the center of the spherical constraint, which the end effector may not violate.

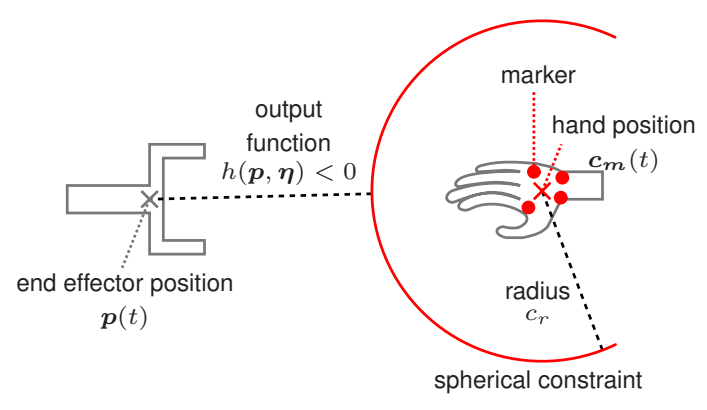

Fig. 5: Experimental setup: The centroid of the markers fitted on a human hand determines the hand position $\boldsymbol{c}_{\boldsymbol{m}}(t)$, which represents the center of the spherical constraint with radius $c_{r}$, constraining the robot end effector.

\section{EXPERIMENTAL EVALUATION}

The characteristics of invariance control are illustrated in the setup depicted in Fig. 4. In the experiment, one constraint is defined, which is given by the human hand. Note that invariance control allows for arbitrarily many constraints and thus it is able to keep multiple humans and obstacles safe and to ensure the avoidance of collisions with the entire volume of the robotic system. By adjusting the forward kinematics, it is also possible to avoid collision with the robot body. The marked human hand is detected by the Qualisys Motion Tracking System. The tracking error amounts to only few $\mathrm{mm}$ and is therefore negligible. The centroid of the rigid body, spanned by the markers, determines the center of a spherical bound with a constant radius. A schematic structure of the experimental setup is shown in Fig. 5. The end effector of an anthropomorphic manipulator with seven degrees of freedom [15] is controlled such that no violation of the spherical constraint occurs. The position of the end effector is calculated using the data from the joint position encoders with 1024 ticks per motor revolution. The invariance controller is implemented in Matlab/Simulink's Real-Time Workshop. The system uses a discrete-time Euler solver, which samples at $1 \mathrm{kHz}$. A JR3 sensor measures the external force $f_{\text {ext }}$ acting on the wrist, which are exerted by the human interaction partner. The nominal controller is an 
impedance controller as introduced in Sec. VI.

\section{A. Boundary Definition}

The constraint limits the translational movement of the end effector in Cartesian space. The task coordinates $\boldsymbol{p}(t) \in \mathbb{R}^{3}$ are given by the end effector position in Cartesian space and the connection to the generalized coordinates is given by the forward kinematics $\boldsymbol{p}=\boldsymbol{f}_{\boldsymbol{p}}(\boldsymbol{q})$ of the manipulator [15]. The constraint is a spherical boundary with a dynamic center position $\boldsymbol{c}_{\boldsymbol{m}}(t) \in \mathbb{R}^{3}$ and a constant radius $c_{r} \in \mathbb{R}$

$$
y=h\left(\boldsymbol{p}(t),\left(\begin{array}{c}
\boldsymbol{c}_{\boldsymbol{m}}(t) \\
c_{r}
\end{array}\right)\right)=c_{r}-\left\|\boldsymbol{p}-\boldsymbol{c}_{\boldsymbol{m}}\right\|_{2} .
$$

In order to enclose an obstacle in this constraint, $\boldsymbol{c}_{\boldsymbol{m}}(t)$ should be chosen as the position of the geometric center of the obstacle and $c_{r}$ has to be large enough so the whole object is contained. Differentiation with respect to time yields

$$
\begin{gathered}
\dot{y}=-\frac{\left(\boldsymbol{p}-\boldsymbol{c}_{\boldsymbol{m}}\right)^{\top}\left(\dot{\boldsymbol{p}}-\dot{\boldsymbol{c}}_{\boldsymbol{m}}\right)}{\left\|\boldsymbol{p}-\boldsymbol{c}_{\boldsymbol{m}}\right\|_{2}} \\
\ddot{y}=-\frac{\left(\boldsymbol{p}-\boldsymbol{c}_{\boldsymbol{m}}\right)^{\top}\left(\ddot{\boldsymbol{p}}-\ddot{\boldsymbol{c}}_{\boldsymbol{m}}\right)+\left\|\dot{\boldsymbol{p}}-\dot{\boldsymbol{c}}_{\boldsymbol{m}}\right\|_{2}^{2}}{\left\|\boldsymbol{p}-\boldsymbol{c}_{\boldsymbol{m}}\right\|_{2}} \\
+\frac{\left(\left(\boldsymbol{p}-\boldsymbol{c}_{\boldsymbol{m}}\right)^{\top}\left(\dot{\boldsymbol{p}}-\dot{\boldsymbol{c}}_{\boldsymbol{m}}\right)\right)^{2}}{\left\|\boldsymbol{p}-\boldsymbol{c}_{\boldsymbol{m}}\right\|_{2}^{3}}
\end{gathered}
$$

Using (5) this yields the I/O-linearized system

$$
z=\ddot{y}=\boldsymbol{a}^{\top} \boldsymbol{f}+b+\beta
$$

with relative degree $r=2$ and

$$
\begin{aligned}
\boldsymbol{a}^{\top}= & -\frac{\left(\boldsymbol{p}-\boldsymbol{c}_{\boldsymbol{m}}\right)^{\top}}{\left\|\boldsymbol{p}-\boldsymbol{c}_{\boldsymbol{m}}\right\|_{2}} \boldsymbol{M}_{\boldsymbol{p}}{ }^{-1} \\
b= & \frac{\left(\boldsymbol{p}-\boldsymbol{c}_{\boldsymbol{m}}\right)^{\top}}{\left\|\boldsymbol{p}-\boldsymbol{c}_{\boldsymbol{m}}\right\|_{2}} \boldsymbol{M}_{\boldsymbol{p}}{ }^{-1}\left(\boldsymbol{C}_{\boldsymbol{p}} \dot{\boldsymbol{p}}+\boldsymbol{g}_{\boldsymbol{p}}\right) \\
& -\frac{\|\dot{\boldsymbol{p}}\|_{2}^{2}}{\left\|\boldsymbol{p}-\boldsymbol{c}_{\boldsymbol{m}}\right\|_{2}}+\frac{\left(\left(\boldsymbol{p}-\boldsymbol{c}_{\boldsymbol{m}}\right)^{\top} \dot{\boldsymbol{p}}\right)^{2}}{\left\|\boldsymbol{p}-\boldsymbol{c}_{\boldsymbol{m}}\right\|_{2}^{3}} \\
\beta= & \frac{\left(\boldsymbol{p}-\boldsymbol{c}_{\boldsymbol{m}}\right)^{\top} \ddot{\boldsymbol{c}}_{\boldsymbol{m}}+2 \dot{\boldsymbol{c}}_{\boldsymbol{m}}^{\top} \dot{\boldsymbol{p}}-\dot{\boldsymbol{c}}_{\boldsymbol{m}}^{\top} \dot{\boldsymbol{c}}_{\boldsymbol{m}}}{\left\|\boldsymbol{p}-\boldsymbol{c}_{\boldsymbol{m}}\right\|_{2}} \\
& +\frac{\left(\left(\boldsymbol{p}-\boldsymbol{c}_{\boldsymbol{m}}\right)^{\top} \dot{\boldsymbol{c}}_{\boldsymbol{m}}\right)^{2}-2\left(\boldsymbol{p}-\boldsymbol{c}_{\boldsymbol{m}}\right)^{\top} \dot{\boldsymbol{c}}_{\boldsymbol{m}}\left(\boldsymbol{p}-\boldsymbol{c}_{\boldsymbol{m}}\right)^{\top} \dot{\boldsymbol{p}}}{\left\|\boldsymbol{p}-\boldsymbol{c}_{\boldsymbol{m}}\right\|_{2}^{3}}
\end{aligned}
$$

Note that $\boldsymbol{a}^{\top}$ and $b$ only depend on the parameters $\boldsymbol{c}_{\boldsymbol{m}}$, while $\beta$ requires the time derivatives of $c_{\boldsymbol{m}}$ up to the second order. Since $\operatorname{dim}(\boldsymbol{u})=\operatorname{dim}(\boldsymbol{f})=3<1=$ no. of constraints holds, (20) is used to determine corrective control. The control loop is similar to Fig. 2 with the difference that the actual robot is replaced by a model. The so-determined desired trajectory is then transmitted to and followed by the actual manipulator. Note that the computation of the control signal is completed during a single sampling interval, thus avoiding any additional time delay in reacting to motions of the human.

\section{B. Experimental Procedure}

During the experiment, the desired trajectory of the end effector $\boldsymbol{p}_{\text {des }}$ is given by a constant desired position. The end effector is held in this position by the impedance controller but shows compliance to forces exerted by the human directly on the end effector. The experiment is carried out twice. During the first run, the marked human hand as shown in Fig. 4 approaches the robot end effector until the manipulator reacts to the approaching boundary and then withdraws again. In the second run, the marked hand approaches while forces are exerted directly to the end effector by a second, unmarked human to show the behavior under bounded disturbance. Both times, the human moves with a velocity up to $0.8 \mathrm{~m} / \mathrm{s}$. The model and boundary parameters are given in Table I.

TABLE I: Experimental parameters

\begin{tabular}{lll}
\hline Cartesian impedance & $\boldsymbol{K}_{p}$ & $600 \mathrm{~N} / \mathrm{m} \cdot \boldsymbol{I}_{3}$ \\
Cartesian damping & $\boldsymbol{D}_{p}$ & $80 \mathrm{~N} \mathrm{~s} / \mathrm{m} \cdot \boldsymbol{I}_{3}$ \\
Mass matrix & $\boldsymbol{M}_{p}$ & $15 \mathrm{~kg} \cdot \boldsymbol{I}_{3}$ \\
Control parameter & $\gamma$ & -18 \\
Desired trajectory & $\boldsymbol{p}_{\mathrm{des}}$ & {$[0.635,0.133,-0.441]^{\top} \mathrm{m}$} \\
Constraint radius & $c_{r}$ & $0.4 \mathrm{~m}$ \\
\hline
\end{tabular}

\section{Experimental Results}

The results of the first run of the experiment are depicted in Fig. 6. Figure 6a shows the deviation of the end effector from the desired trajectory $\boldsymbol{p}_{\mathrm{des}}$ and the value of the invariance function (14). The system deviates from the desired trajectory, whenever a constraint approaches and the trajectory lies outside of the admissible set, i.e. the invariance function approaches zero. As soon as the constraint is removed, i.e. the invariance function decreases, the deviation is reduced to zero. This gives an indication for the stability of the controlled system. The figure also shows that the invariance function is reduced to zero and never takes a positive value apart from a slight chattering effect due to the sampled time implementation. This indicates the adherence to the constraints and the positive invariance of the system. The invariance is also emphasized by Fig. 6b, which shows that the condition for invariance (25) is fulfilled, since

$$
z_{c}=\gamma \leq b+\beta
$$

holds for all instants of time.

The forces exerted during the second run of the experiment in direction of the boundary are depicted in Fig. 7a. Figure $7 b$ shows the influence of the forces on the deviation from the desired trajectory and on the invariance function. As expected, the forces cause a deviation from the desired trajectory due to the nominal impedance controller as observable during the first $10 \mathrm{~s}$ of Fig. 7b. They have, however, no effect on the invariance of the system. Forces directed away from the boundary as for example at $t=25 \mathrm{~s}$ result in compliant behavior and a decrease in the invariance function. Forces towards the boundary, on the other hand, do not result in a violation of the constraint, as it is visible at $t=30 \mathrm{~s}$ since the invariance controller causes a stiff behavior. Additionally, in this run the condition for invariance (25) is also fulfilled as shown in Fig. 7c. These experimental results encourage the use of invariance control with robotic manipulators and constraints with dynamic parameters. 


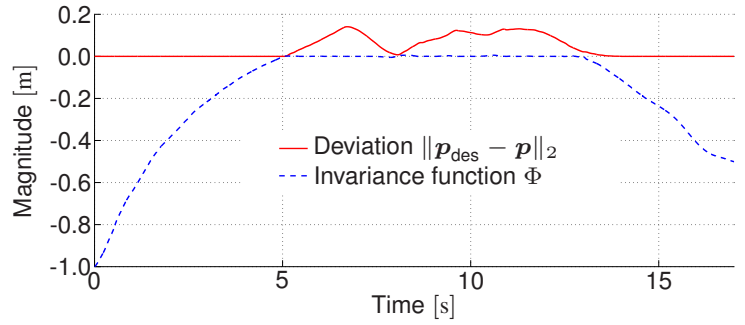

(a)

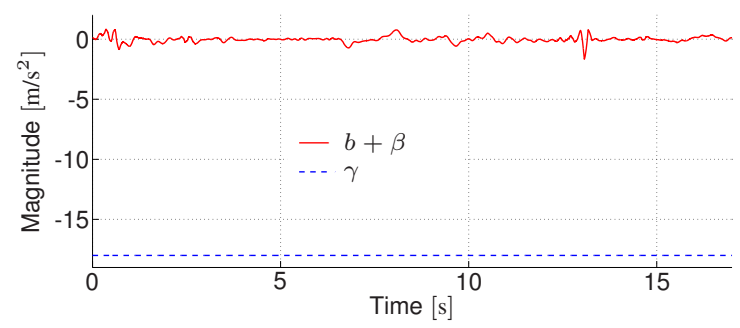

(b)

Fig. 6: Evaluation of (a) the deviation from the desired trajectory and the invariance function value and (b) the invariance condition (34) in the absence of external forces

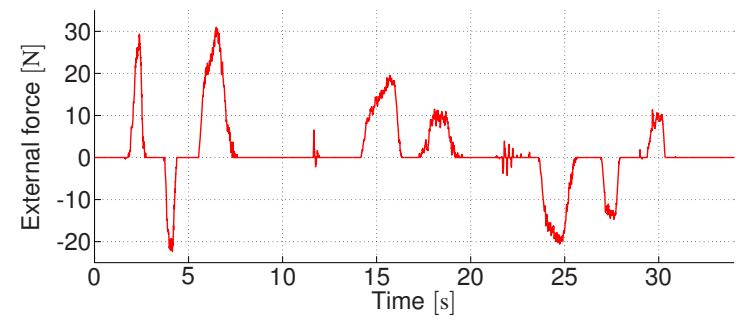

(a)

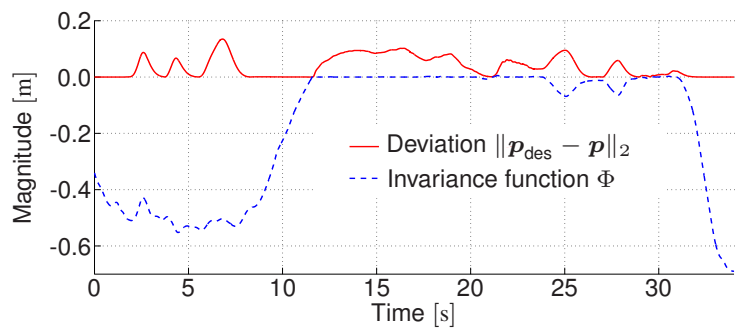

(b)

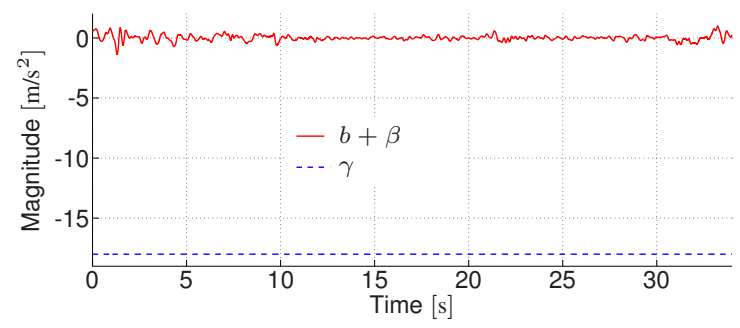

(c)

Fig. 7: Influence of (a) the magnitude of the external forces towards the constraint (positive towards the constraint) on (b) the deviation from the desired trajectory, the invariance function value and (c) the condition (34)

\section{CONCLUSION}

Aiming for safety in human-robot interaction scenarios, a novel approach for controlling a robot manipulator in an environment with dynamic constraints is introduced. The method extends the invariance control framework to allow for constraints with dynamic parameters. The extension preserves the stability of the controlled system. Furthermore, it renders the robotic manipulator invariant with respect to the dynamic constraints if the constraint dynamics are sufficiently often continuously differentiable and bounded with bounded derivatives. Results of the experiment involving the interaction with a human show the effective compliance with the constraints even under the presence of external forces.

\section{ACKNOWLEDGEMENTS}

The research leading to these results has received funding from the European Union Seventh Framework Programme FP7/2007-2013 within the ERC Starting Grant Control based on Human Models (con-humo) under grant agreement no. 337654 .

\section{REFERENCES}

[1] E. Rimon and D. Koditschek, "Exact Robot Navigation Using Artificial Potential Functions," IEEE Transactions on Robotics and Automation, vol. 8, no. 5, pp. 501-518, 1992.

[2] L. Rosenberg, "Virtual Fixtures: Perceptual Tools for Telerobotic Manipulation," in Proceedings of the IEEE Virtual Reality Annual International Symposium, 1993, pp. 76-82.

[3] D. Mayne, J. Rawlings, C. Rao, and P. Scokaert, "Constrained model predictive control: Stability and optimality," Automatica, vol. 36, no. 6, pp. 789-814, 2000.

[4] A. Ames, J. Grizzle, and P. Tabuada, "Control Barrier Function based Quadratic Programs with Application to Adaptive Cruise Control," in IEEE 53rd Annual Conference on Decision and Control (CDC), Dec 2014, pp. 6271-6278.

[5] E. Gilbert and I. Kolmanovsky, "Nonlinear tracking control in the presence of state and control constraints: a generalized reference governor," Automatica, vol. 38, no. 12, pp. 2063-2073, 2002.

[6] J. Mareczek, M. Buss, and G. Schmidt, "Sufficient Conditions for Invariance Control of a Class of Nonlinear Systems," in 39th IEEE Conference on Decision and Control, Sydney, Australia, Dec. 2000, pp. 1436-1442.

[7] M. Scheint, J. Wolff, and M. Buss, "Invariance Control in Robotic Applications: Trajectory Supervision and Haptic Rendering," in American Control Conference (ACC), Seattle, USA, 2008, pp. 1436-1442.

[8] J. Wolff and M. Buss, "Invariance Control Design for Nonlinear Control Affine Systems under Hard State Constraints," in NOLCOS'2004 Symposium on Nonlinear Control Systems, Stuttgart, Germany, Sept. 2004, pp. 711-716.

[9] _- "Invariance Control Design for Constrained Nonlinear Systems," in 16th IFAC World Congress, Prague, Czech Republic, July 2005.

[10] M. Kimmel, M. Lawitzky, and S. Hirche, "6D Workspace Constraints for Physical Human-Robot Interaction using Invariance Control with Chattering Reduction," in IEEE/RSJ International Conference on Intelligent Robots and Systems (IROS), 2012, pp. 3377-3383.

[11] A. Isidori, Nonlinear Control Systems, 3rd ed. Springer-Verlag, Berlin, 1995.

[12] H. Khalil, Nonlinear Systems, 3rd ed. Prentice Hall, 1996.

[13] M. Kimmel and S. Hirche, "Invariance Control with Chattering Reduction," in IEEE 53rd Annual Conference on Decision and Control (CDC), 2014.

[14] A. Albu-Schäffer and G. Hirzinger, "Cartesian Impedance Control Techniques for Torque Controlled Light-Weight Robots," in Proceedings of the IEEE International Conference on Robotics and Automation, vol. 1, 2002, pp. 657-663.

[15] B. Stanczyk and M. Buss, "Development of a Telerobotic System for Exploration of Hazardous Environments," in Proc. IEEE/RSJ IROS, 2004, pp. 2532-2537. 